\title{
Transcriptome Analysis of gene in Mouse M-1 cells revealing the functional mechanism of Astragali radix extract
}

\section{Bing Wang}

ENNOVA Institute of Life Science and Technology

\section{Qian Feng}

ENNOVA Institute of Life Science and Technology

chao zhang

ENNOVA Institute of Life Science and Technology

\section{Yuming Chen}

ENNOVA Institute of Life Science and Technology

\section{Yu Chen}

ENNOVA Institute of Life Science and Technology

Jie Teng

ENNOVA Institute of Life Science and Technology

\section{Zhongzhen Cai}

ENNOVA Institute of Life Science and Technology

\section{Mengmei Li}

ENNOVA Institute of Life Science and Technology

\section{Lin Wang}

ENNOVA Institute of Life Science and Technology

\section{Yanpeng Zhao}

ENNOVA Institute of Life Science and Technology

Xuemei Bai ( $\sim$ baixuemei@enn.cn )

ENNOVA Institute of Life Science and Technology

\section{Research}

Keywords: Astragali Radix, RNA-Seq, Qi, mitochondria, M-1

Posted Date: September 15th, 2021

DOI: https://doi.org/10.21203/rs.3.rs-889398/v1 
License: (c) (i) This work is licensed under a Creative Commons Attribution 4.0 International License. Read Full License

Version of Record: A version of this preprint was published at Pharmacology \&amp; Pharmacy on January 1st, 2021. See the published version at https://doi.org/10.4236/pp.2021.1212025. 


\section{Abstract \\ Background}

Astragali Radix (AR),the dried root of legumes, belongs to the Qi- invigorating herbs in traditional Chinese medicine and plays an important role in the treatment of many diseases. In order to understand the mechanism of action of AR extract, we used AR extract to treat M-1, mouse kidney cells, and used transcriptome sequencing technology to detect the genomic transcription level of the cells under the action of AR at different concentrations and times.

\section{Result}

The results showed that after a low concentration of AR treatments on the cells, the expression of genes related to cell growth and cellular immune response changed significantly, among which multiple genes are related to mitochondrial function, while high concentrations of AR affected the expression of histones and disease-related genes. It showed that the low concentration of AR extract can achieve the effect of invigorating Qi by regulating the function of mitochondria. In addition, several important genes and pathways were identified as potential targets of AR activation.

\section{Conclusion}

The research not only clarified the main molecular biological mechanism of AR invigorating Qi, but also provided experimental basis and cellular physiology reference for the further clinical application of AR.

\section{Background}

Astragali Radix (AR), a perennial leguminous herb whose root used as medicine, is one of the most common and popular Chinese herbal medicines. In more than a thousand years of the development of the traditional Chinese medicine, people have discovered many drugs for invigorating Qi, and AR is a representative one for Qi invigoration. Based on the important role of AR, a variety of AR medicinal products have been developed, include Astragalus tablets, Astragalus Polysaccharide Granules, Astragalus Glucoside Granules, etc., which have been used in the treatment of many diseases, including COVID-19, a novel coronavirus pneumonia pandemic, occurred in early 2020 . The combined use of Astragalus and other Chinese herbal medicines played an important role in the prevention and treatment of COVID-19 infections [1], saving many lives and reducing economic losses.

A variety of natural plant products can be extracted from AR, mainly containing Astragaloside, Astragalus flavonoids, Astragalus polysaccharides (APS) and amino acids. Astragaloside IV can significantly reduce myocardial damage caused by ischemia and hypoxia, improve energy metabolism, improve endothelial dysfunction, promote angiogenesis, improve immune function, and regulate blood sugar and blood lipid 
levels [2]. Flavonoids are mainly ingested as glycosides, which have exhibited many potential beneficial activities in animal research and human trials [3]. APS is a type of water-soluble heteropolysaccharide with biological activity, which has the characteristics of low toxic and side effects, no residue and intolerance. The biological functions of APS include regulating immune function, anti-aging, anti-tumor [4], lowering blood sugar, lowering blood fat, anti-fibrosis, antibacterial, radiation protection and antiviral effects [5]. AR extract was the isolated effective ingredients from AR dry root, and its biological effects was as effective as dry AR root.

In the literature, the existing high-throughput sequencing studies related to AR are either the analysis of the therapeutic effects of the main components of AR on disease models [6, 7], or the analysis of the effects of AR extracts on plants development [8]. Except for the transcriptome analysis of compound medicines containing AR [9], there is no transcriptome analysis research on the effect of AR extract to illustrate the healing effect of AR. For this study, we applied the extract of AR, a traditional Chinese medicine for invigorating Qi, to mouse M-1 cells, and used modern transcriptomics methods to screen differently expression genes during cell culture to study the effects of traditional Chinese medicine on cells. It is a bridge between traditional medicine and modern medical research, which helps us to understand the functional mechanism of the Qi-invigorating herb.

\section{Result}

\section{Transcriptome analysis of the effect of low-dose AR on M-1 cell}

In order to reveal the molecular mechanism of the effects of AR on cells after different periods of exposure time, the low-dose AR extract $(50 \mu \mathrm{g} / \mathrm{ml})$ was added to the cell culture medium, and the cell pellets were collected at two time points of $4 \mathrm{~h}$ and $24 \mathrm{~h}$. Use high-throughput sequencing to remove predicted genes (gene names start with GM and then coded with numbers), and compare gene expression changes between the control group and treatment group at the same time point. The results showed that 439 Differently expressed genes (DEGs) (including 233 up-regulated genes and 206 down-regulated genes) and 475 (including 268 up-regulated genes and 207 down-regulated genes) were screened out between $4 \mathrm{~h}$ and $24 \mathrm{~h}$ after AR treatment (Ifold change $>2$ and $p<0.05)$ (Fig. 1, A,B). The result showed that the AR extract made a greater impact on cell growth after AR treating the cells.

So as to understand the mechanism of AR more clearly, we chose to analyze the 64 common differentially expression genes(Co-DEGs) (Fig. 1C). Gene Ontology (GO) analysis classifies and enriches differential genes from three different dimensions: Biological Process(BP), Cellular Component(CC), and Molecular Function (MF), thereby to reflect the dynamic change process between different groups. It was found that there were more enriched genes in biological regulation, metabolic process and cell development in BP term. The cell part, organelle part, membrane and other enriched genes in CC term were more abundant, and the binding, catalytic activity, molecular function regulation and other enriched genes in MF term were more enriched. The most enriched BP terms included response to UV-A, synthesis 
and regulation of $\mathrm{MHC}$ class II biosynthetic processes ( $T / r 4$, Spi1), response to toxic substances, immune response (Spi1, C3, Rnf26, II2, T/r4) and protein modification. The number of enriched genes related to protein modification was the largest among them. Receptor complex (Adrb3, TIr4, Itga 10) and cilium were the most enriched CC terms. The most enriched MF terms were growth factor activity (Cers 1, Tgfa, II2), carbohydrate transmembrane transporter activity (SIc2a4, Aqp3) (Fig. 1, D-G). It showed that the AR extract had a greater regulatory effect on cell growth and immune response-related genes after AR acting on cells.

Kyoto Encyclopedia of Genes and Genomes (KEGG) pathway enrichment analysis was performed to explore the most significantly enriched DEG pathway (Fig. 1, H). Among the top 20 KEGG pathways, the most enriched were Chagas disease (American trypanosomiasis), NOD-like receptor signaling pathway (Nek7, Antxr1, TIr4), PI3K-AKT signaling pathway (II2, Tgfa, TIr4, Itga10 ), HTLV-I infection(Spi1, II2, RasI29), and pathways in cancer. NOD-like receptor signaling pathway played a key role in pathogen recognition and innate immune response; PI3K-AKT signaling pathway was involved in the regulation of many basic cellular processes, indicating that the effects of AR extract on cells changed cellular immunerelated genes.

\section{Transcriptome analysis of the effect of high-dose AR on M- 1 cell}

The effects of high-dose AR extract on M-1 cells were studied, in which the concentration of AR was increased to 5 times the original $(250 \mu \mathrm{g} / \mathrm{ml})$. As in the previous experiment, the cells were cultured for 4 hours with AR extract, and then the cells were harvested. By comparing the gene expression changes between the control group and treatment group, DEGs were analyzed to identify the effects of highconcentration AR extract on the cells. The experimental results showed that a total of 64 DEGs were detected, of which 35 genes were up-regulated and 29 genes were down-regulated (|fold change|>2 and $p$ $<0.05$ ) (Fig. 2, A). The result revealed that the high-concentration AR extract had an significant effect on cell growth after treating the cells.

In order to clarify the effective mechanism of the high concentration of AR extract on the growth of cells, the 64 DEGs that were screened were further analyzed. GO analysis showed that BP was mainly concentrated in DNA, nucleosome assembly, negative regulation of immune system process and $\mathrm{T}$ cell migration. CC terms were mainly enriched in the intrinsic component of plasma membrane, integral component of plasma membrane, and intrinsic component of membrane. The MF terms were enriched in gated channel activity, and protein heterodimerization activity (Fig. 2, B-D). It showed that the high concentration of AR extract mainly acted on basic life processes such as DNA assembly and material transmembrane transportation. Changes in these processes were more likely to change the cell growth state.

KEGG pathway enrichment analysis of differential genes was performed, and the results showed that among the top 20 signaling pathways, the most enriched KEGG pathways were related to diseases such as alcohol abuse, systemic lupus erythematosus, renin secretion, viral carcinogenesis, taste transmission, 
and Shigellosis (Fig. 2, E). It was also found that the genes enriched in the pathways of alcoholism and Systemic lupus erythematosus were all histones (Table 1). As it was known, histones was involved in the assembly of nucleosomes[10], and histone modifications were the main type of epigenetic modification. Histones were modified by a large number of enzymes and related to a variety of cancers $[11,12]$. The results showed that AR extract affected the gene expression of histones genes and different concentration of AR had different effect effects, which indicated that AR extract affected the assembly of nucleosomes in multiple manners, which caused the different clinic functions of AR. We performed Venn analysis on the DEGs in the same time period of high dose and low dose, and found a total of 17 CoDEGs (Table 2). 8 co-DEGs (2810468N07Rik, II27ra, Dnah5, AC123954.1, Pcdhgc4, Gp1bb, Clcn1, Ccl20) were found to have opposite expression levels in the two groups. The genes expression of high dose AR among these co-DEGs were positively correlated with the occurrence of diseases, which demonstrated that the KEGG pathways altered by high-concentration AR extract were related to the occurrence of diseases, and had side effects on cells growth. 
Table 1

Top 20 pathways of KEGG enrichment

\begin{tabular}{|c|c|c|c|}
\hline ID & Descrption & $\begin{array}{l}\text { DEGs } \\
\text { number }\end{array}$ & Genes \\
\hline ko05034 & Alcoholism & 4 & Hist1h4c,Hist1h4d,Hist2h3b,Hist2h2bb \\
\hline ko05322 & Systemic lupus erythematosus & 4 & Hist1h4c,Hist1h4d,Hist2h3b,Hist2h2bb \\
\hline ko04924 & Renin secretion & 2 & Clca1,Clca3a1 \\
\hline ko05203 & Viral carcinogenesis & 3 & Hist1h4c,Hist1h4d,Hist2h2bb \\
\hline ko04742 & Taste transduction & 2 & Scnn1g,Tas1r1 \\
\hline ko05131 & Shigellosis & 3 & Myl7,Gjb2,Hist2h3b \\
\hline ko04972 & Pancreatic secretion & 2 & Clca1,Clca3a1 \\
\hline ko00910 & Nitrogen metabolism & 1 & Car15 \\
\hline ko00512 & Mucin type 0-glycan biosynthesis & 1 & B3gnt6 \\
\hline ko04960 & $\begin{array}{l}\text { Aldosterone-regulated sodium } \\
\text { reabsorption }\end{array}$ & 1 & Scnn1g \\
\hline ko04640 & Hematopoietic cell lineage & 2 & Gp1bb,Cd55b \\
\hline ko02010 & $\mathrm{ABC}$ transporters & 1 & Abca4 \\
\hline ko04060 & $\begin{array}{l}\text { Cytokine-cytokine receptor } \\
\text { interaction }\end{array}$ & 2 & II27ra,Ccl20 \\
\hline ko04512 & ECM-receptor interaction & 1 & Gp1bb \\
\hline ko04610 & $\begin{array}{l}\text { Complement and coagulation } \\
\text { cascades }\end{array}$ & 1 & Cd55b \\
\hline ko04061 & $\begin{array}{l}\text { Viral protein interaction with } \\
\text { cytokine and cytokine receptor }\end{array}$ & 1 & $\mathrm{Ccl} 20$ \\
\hline ko04657 & IL-17 signaling pathway & 1 & $\mathrm{Ccl} 20$ \\
\hline ko04928 & $\begin{array}{l}\text { Parathyroid hormone synthesis, } \\
\text { secretion and action }\end{array}$ & 1 & Mmp17 \\
\hline ko04670 & $\begin{array}{l}\text { Leukocyte transendothelial } \\
\text { migration }\end{array}$ & 1 & Myl7 \\
\hline ko04668 & TNF signaling pathway & 1 & $\mathrm{Ccl} 20$ \\
\hline
\end{tabular}


Table 2

co-DEGs of low and high dose AR in M-1

\begin{tabular}{|llll|}
\hline GENE NAME & GENE ID & AT04 vs ACO4 L & AT04 vs AC04 H \\
\hline Prnd & ENSMUSG00000027338 & 12.49022414 & 2.935376615 \\
\hline U90926 & ENSMUSG00000029409 & 12.01272928 & 12.55300364 \\
\hline 2810468N07Rik & ENSMUSG00000091475 & 10.95329434 & -2.664937743 \\
\hline II27ra & ENSMUSG00000005465 & 9.865114619 & -9.376436547 \\
\hline Mmp17 & ENSMUSG00000029436 & 8.970767394 & 8.159787261 \\
\hline Dnah5 & ENSMUSG00000022262 & 7.333065878 & -2.225834753 \\
\hline Gpa33 & ENSMUSG00000000544 & 2.512943944 & 2.065910967 \\
\hline AC123954.1 & ENSMUSG00000113255 & -2.026644561 & 2.080883822 \\
\hline Pcdhgc4 & ENSMUSG00000023036 & -2.094222386 & 2.330155403 \\
\hline Rnf26 & ENSMUSG00000053128 & -5.923689501 & -5.339661043 \\
\hline Gp1bb & ENSMUSG00000050761 & -9.056897033 & 5.74293525 \\
\hline Clcn1 & ENSMUSG00000029862 & -9.577372177 & 9.583446374 \\
\hline A430105J06Rik & ENSMUSG00000097784 & -11.07984475 & -11.01433669 \\
\hline Cd55b & ENSMUSG00000026401 & -11.67142607 & -11.22068868 \\
\hline Prrx2 & ENSMUSG00000039476 & -11.68359558 & -3.52890236 \\
\hline Cc120 & ENSMUSG00000026166 & -11.86405843 & 3.292019206 \\
\hline A530084C06Rik & ENSMUSG00000090863 & -12.11355661 & -10.13644007 \\
\hline
\end{tabular}

\section{Discussion}

AR is a traditional Chinese medicine for invigorating Qi that is widely used in clinical Chinese medicine, and its clinical efficacy has been universally recognized by modern medicine [13]. Qi is a relatively abstract concept in traditional Chinese medicine. The theory of traditional Chinese medicine believes that Qi is the driving force of human life activities and one of the basic substances that maintain the normal life activities of the human body from different aspects. Studies have shown that AR extract has complex components, including Astragaloside, Astragalus flavonoids, APS and amino acids, which play a role in immune regulation, multi-organ protection, hypoglycemia, anti-virus, and anti-tumor $[13,14]$. As so far, the cellular biological mechanism of AR curative effect is not clear.

In order to understand the AR effects on cells through modern technology, we used AR extract to treat cells for different times and concentrations. With the help of transcriptomics technology, we analyzed the 
changes in cell gene transcription levels and reveal the significance of AR in cell life activities. The results showed that treatment of cells with a low concentration of AR extract can screen out prolific DEGs, and the co-DEGs of different treatment times were mainly enriched in cell growth, metabolic processes, immune response and other related pathways, which were consistent with the multiple clinical function of AR in Chinese medicine [13]. After a high dose of AR acts on cells, its signal pathways are mainly concentrated on disease-related pathways such as systemic lupus erythematosus, viral carcinogenesis, and Shigellosis. The results well explained that the high dose of AR extract may cause some side-effect in healthy people.

The growth and development of cells is usually a complex process involving various regulatory molecules. Cell growth depends on the comprehensive effects of biochemical and molecular processes that synthesize its various components inside the cell. In our previous experimental results, it was found that low dose of AR increased the content of ATP and GSH in cells [15]. ATP is the carrier of energy released by cells during respiration and directly provides energy for various life activities. Mitochondrial homeostasis is important for maintaining mitochondrial function and cell stability [16]. Low concentration of AR acted on the differential genes screened by cells, and multiple genes become potential target genes, such as ceramide synthase 1(Cers1), aquaporin 3 (Aqp3), Interleukin-2 (II2) and so on (Result 3.1). Cers1 is a transduction stress signal that regulates lipid metabolism from the endoplasmic reticulum to the outer mitochondrial membrane [17]. The transmembrane transport of $\mathrm{H}_{2} \mathrm{O}_{2}$ requires the participation of $A q p . \mathrm{H}_{2} \mathrm{O}_{2}$ crosses the cytoplasmic membrane from outside the cell into the cytoplasm with the assistance of Aqp3 [18]. Aqp3 can also inhibit cell apoptosis by reducing the genes expression related Wnt/GSK-3 $\beta / \beta$-catenin pathway. Toll-like receptor $4(T / r 4)$ participates in a variety of autoimmune diseases by acting as a bridge between innate immunity and acquired immunity. The activation of T/r4 induces mitochondrial dynamic imbalance and promotes the occurrence of diseases [19]. NIMA-related kinase 7 (Nek 7 ) is a multifunctional kinase that affects centrosome replication, mitochondrial regulation, intracellular protein transport, DNA repair and mitotic spindle assembly [20], and participates in NF-KB signaling. (I/2) is a pro-inflammatory cytokine that can regulate cell growth, proliferation, and apoptosis. After II2 stimulation, the quality of mitochondria changes, or the production of ROS in cells increases [21, 22]. In addition, there are some differential gene functions related to mitochondria, such as the perredoxin ( Prx) family that controls the level of $\mathrm{H}_{2} \mathrm{O}_{2}$ in the cell and regulates signal transduction [23]. Glutathione S-transferase (GST) family members 7 (Gstm7) exerts the detoxification effect of electrophilic compounds by binding to reduced glutathione [24]. The transcription of E3 ligase proteins Fbxo15 and Ring finger protein 26 (Rnf26) is involved in the immune regulation function of cells $[25,26]$. These genes are directly and indirectly related to the function of mitochondria. And it can be seen from Fig. 1, E that in the BP of co-DEGs, protein modification related genes had the most enrichment. It showed that low concentrations of AR may play a role in changing the function of mitochondria by modifying the protein after expression of mitochondrial-related genes, thereby improving cellular immune function and cell growth. 
The decrease of mitochondrial membrane potential can cause a series of biochemical changes in the mitochondrial membrane, which is an early event in the process of cell apoptosis [27]. High concentration of AR reduces the production of ATP and lowers the membrane potential of mitochondria [15]. Excessive mitochondrial fission can cause mitochondrial ROS overload and inhibit the production of mitochondrial ATP [28]. As it showed in Table 1, the high concentration of AR extract produced drug toxicity for cell growth and caused cellular immune response. Among the co-DEGs shared with the low-concentration AR extract, the expression levels of multiple genes showed opposite results (Table 2). The expression patterns of these genes under the action of high concentrations of AR were mostly related to the occurrence of diseases. Studies have found that human neutrophils treated with Interleukin-27 (II27) swelled the cells, ruptured mitochondria and condensed the nucleus [29]. Chemokine (C-C motif) ligand 20 (CCL20) is expressed in various human tissues and immune cells, and involved in local immune cell recruitment [30]. CCL20 is significantly up-regulated in a variety of cancer tissues. CCL20 produced by liver cancer cells recruits CCR6 + CD $5+B$ cells and induces blood vessels generated to promote tumor growth [31]. In our experiments, it was also found that $C c / 20$ expression was highly expressed under the action of high concentrations of AR. The mutation of $C / c n 1$ is related to myotonia [32]. GPIb-IX-V complex is expressed on megakaryocytes and platelets, which acts as a receptor for von Willebrand factor and mediates platelet-subendothelial interactions, and is a major participant in hemostasis. The glycoprotein Ib (GPIb) -IX-V complex contains four different transmembrane subunits: GPIba, GPIb $\beta$, GPIX and GPV. Mutations in GPIb $\beta$ caused GPIb-IX-V defects [33]. Some experiments have found that high concentrations of AR can shorten the lifespan of bees and also have an inhibitory effect on the growth of cancer cells [34,35]. It was obvious that high concentrations of drugs had adverse effects on cells and may promote the occurrence of diseases. In addition, the high dose of AR extract also changes the expression of multiple histones, which can cause some genes to change their expression patterns at the DNA level. This also shows that the high concentration of AR extract may have drug toxicity on cells, resulting in changes in gene expression patterns related to diseases.

Based on the RNA-Seq analysis, we gained further understanding of the molecular mechanism of AR, and also learned the preliminary functional mechanism for the side effects of the high drug concentration AR. Several genes were identified as potential targets of $A R$, which were expected to become treatment strategies for diseases caused by abnormal expression of the them. Our future research will focus on animal model to verify these hypotheses.

\section{Conclusion}

Using RNA-Seq, the DEGs related to the effects of the AR extract on the cells were identified. The GO and KEGG function enrichment analysis of DEGs showed that a low concentration of AR extract was beneficial to cell growth and enhance the expression of genes related to cellular immune response. Many genes are related to mitochondrial function, and high concentrations of AR may cause abnormal expression of multiple disease-related genes in cells. Here, for the first time, we use AR extract to directly act on healthy cells, and explain the role of AR from the perspective of mitochondria. This results have a 
guiding role in accurately understanding the pharmacological effects of AR and its molecular mechanism, which is helpful for application of AR extract in disease prevention and treatment.

\section{Materials And Methods \\ Extraction of Astragali Radix}

Astragalus root of 50 grams (g) was sliced to pieces and powdered. The powder was soaked in $500 \mathrm{ml}$ distilled water for 1 hour (h) and then boiled at $100^{\circ} \mathrm{C}$ for 30 minutes. The mixed liquid was filtered to get the supernatant, and the insoluble matter was again mixed with $400 \mathrm{ml}$ distilled water and boiled at 100 ${ }^{\circ} \mathrm{C}$ for 20 minutes. The supernatant was obtained by the same way and mixed with the former supernatant. The mixture was concentrated by rotary evaporation apparatus to obtain approximate $7.2 \mathrm{~g}$ of extracted product.

\section{Cells culture and treatment}

Mouse cell line M1 (ATCC, CRL-2038) was grown in Dulbecco's modified Eagle's medium (DMEM) supplemented with $10 \%$ fetal bovine serum (FBS) and $1 \%$ penicillin-streptomycin. Cells were cultured at $37^{\circ} \mathrm{C}$ in a $5 \% \mathrm{CO}_{2}$ incubator. During the experimental period, cells were divided into three groups, which were low dose, high dose and control group (CK), respectively. Low dose extraction of Astragalus (50 $\mu \mathrm{g} / \mathrm{ml}$ ) was added to the supernatant of cells and incubated for $4 \mathrm{~h}$ and $24 \mathrm{~h}$ respectively, and then the supernatant was discarded. High dose extraction of Astragalus $(250 \mu \mathrm{g} / \mathrm{ml})$ was added to the supernatant of cells and incubated for $4 \mathrm{~h}$, followed by removing of AR supernatant. Cells with no AR treatment were used as controls, which were processed with the same methods except that same volume of PBS was added instead of AR extraction. The cells were trypsinized, washed with PBS, and the cell pellet was collected and washed with PBS again. The experiment was conducted in three biological replicates.

\section{RNA extraction and construction of cDNA library}

Total RNA of M1 cells treated with extraction of Astragalus or mock treated was extracted and isolated using TaKaRa MiniBEST Universal RNA Extraction Kit (9767) according to the manufacturer's instructions. The quantity and purity of RNA was assessed by Agarose gel electrophoresis.

RNA was then sent to Sangon Biotech (Shanghai) Co., Ltd for transcriptome analysis. mRNA was isolated from total RNA and then reverse transcribed into cDNA to construct the cDNA library. Sequences were initial analyzed using Illumina HiSeqTM system, Shanghai Sangon Biotech, Shanghai, China.

\section{Library quality identification and sequence comparison analysis}

The quality of raw data, sequences obtained from the Illumina HiSeqTM system, were further assessed by FastQC software. Nucleic acid sequences containing low quality base, unknown base, etc. were 
excluded. The remaining sequences after quality control were compared with the reference genome of Mus musculus. Expression abundance of genes was calculated in terms of transcripts per million (TPM). Data were obtained form 3 independent biological replicates.

\section{Screening of differentially expressed genes}

DEGs were analyzed by DEGseq software. Genes with $p$ value $<0.05$ and $\log _{2}$ (fold change) $>2$ were considered significantly differently expressed. Significant DEGs in all groups were calculated and shown in Volcano and Veen map. The overlap of Veen map represented the common DEGs between groups and indicated the connection of DEGs from each group. Numbers in Veen map represented the unique or universal DEGs between groups.

\section{Analysis of GO and KEGG}

The molecular function, subordinate cellular component and involved biological process of DEGs were analyzed by GO. KEGG database were used for functional annotation of the DEGs.

\section{Abbreviations}

AR Astragali Radix

APS Astragalus polysaccharides

DEGs Differently expressed genes

Co-DEGs Common differentially expression genes

GO Gene Ontology

KEGG Kyoto encyclopedia of genes and genomes

BP Biological process

CC Cellular component

MF Molecular function

Cers 1 Ceramide synthase 1

Aqp Aquaporins

TIr4 Toll-like receptor 4

II2 Interleukin-2

Nek7 NIMA-related kinase 7 
Prx Perredoxin

Gstm7 Glutathione S-transferase (GST) family members 7

Ccl20 Chemokine (C-C motif) ligand 20

Rnf26 Ring finger protein 26

II27 Interleukin-27

GPIb Glycoprotein Ib

\section{Declarations}

\section{Ethics approval and consent to participate}

Not applicable.

\section{Consent for publication}

Not applicable.

\section{Availability of data and materials}

The datasets used and/or analysed during the current study are available from the corresponding author on reasonable request.

\section{Competing interests}

The authors have no competing interest to report.

\section{Funding}

ENN Research Fund

\section{Authors' contributions}

BW designed the specific plan of the experiment and the modification of the article; QF performed cell culture experiment and RNA extraction; CZ was a major contributor in analysising data and writing the manuscript; YC wrote the first draft of the article; ML extracted astragalus Radix; YC and LW was responsible for the drug concentration exploration before the experiment; JT was assisted in data analysis; ZC was responsible for the reference review; YZ assisted in RNA extraction; XB provided conceptual contributions, overall design and guidance. All authors read and approved the final manuscript. 
Authors are arranged in order of contribution. *Both authors (Bing Wang \& Qian Feng) contributed equally to this work, and they are both the first author. ${ }^{\#}$ Corresponding author.

\section{Acknowledgements}

We thank the ENN Group for providing the funding for this research. Bioinformatic analysis was performed using the OmicShare tools, a free online platform for data analysis. We are also grateful to the editor and anonymous reviewers for their constructive criticisms and suggestions, which significantly improved this manuscript.

\section{References}

1. Adhikari B, Marasini BP, Rayamajhee B, et al. Potential roles of medicinal plants for the treatment of viral diseases focusing on COVID-19: A review. Phytother Res. 2021;35(3):1298-312.

2. Tan YQ, Chen HW, Li J, Astragaloside IV. An Effective Drug for the Treatment of Cardiovascular Diseases. Drug Des Devel Ther. 2020;14:3731-46.

3. Hostetler GL, Ralston RA, Schwartz SJ, Flavones: Food Sources, Bioavailability, Metabolism, and Bioactivity. Adv Nutr, 2017. 8(3): 423 - 35.

4. Wu CY, Ke Y, Zeng YF, et al. Anticancer activity of Astragalus polysaccharide in human non-small cell lung cancer cells. Cancer Cell Int. 2017;17:115.

5. Zheng Y, Ren W, Zhang L, et al. A Review of the Pharmacological Action of Astragalus Polysaccharide. Front Pharmacol. 2020;11:349.

6. Dong N, Li X, Xue C, et al. Astragalus polysaccharides alleviates LPS-induced inflammation via the NF-kappaB/MAPK signaling pathway. J Cell Physiol. 2020. doi:10.1155/2020/9542165.

7. Zhang Y, Tao C, Xuan C, et al., Transcriptomic Analysis Reveals the Protection of Astragaloside IV against Diabetic Nephropathy by Modulating Inflammation. Oxid Med Cell Longev, 2020. 2020: 9542165.

8. Mhiri W, Ceylan M, Turgut-Kara N, et al. Transcriptomic analysis reveals responses to Cycloastragenol in Arabidopsis thaliana. PLoS One. 2020. doi:10.1371/journal.pone.0242986.

9. Lu T, Bian Y, Zhu Y, et al. HUANGKUISIWUFANG inhibits pyruvate dehydrogenase to improve glomerular injury in anti-Thy1 nephritis model. J Ethnopharmacol. 2020;253:112682.

10. Fyodorov DV, Zhou BR, Skoultchi Al, et al. Emerging roles of linker histones in regulating chromatin structure and function. Nat Rev Mol Cell Biol. 2018;19(3):192-206.

11. Xie W, Zhang J, Zhong P, et al. Expression and potential prognostic value of histone family gene signature in breast cancer. Exp Ther Med. 2019;18(6):4893-903.

12. Karsli-Ceppioglu S, Dagdemir A, Judes G, et al. Epigenetic mechanisms of breast cancer: an update of the current knowledge. Epigenomics. 2014;6(6):651-64.

13. Zhang $\mathrm{CH}$, Yang X, Wei JR, et al. Ethnopharmacology, Phytochemistry, Pharmacology, Toxicology and Clinical Applications of Radix Astragali. Chin J Integr Med. 2021;27(3):229-40. 
14. Liu P, Zhao H, Luo Y. Anti-Aging Implications of Astragalus Membranaceus (Huangqi): A Well-Known Chinese Tonic. Aging Dis. 2017;8(6):868-86.

15. Chen Y, Feng Q, Li M, et al. The Effect of TCM Herbs on Mitochondrial Functions: The Linkage between Qi and Mitochondria. Chinese Medicine. 2020;11(04):127-41.

16. Youle RJ, van der Bliek AM. Mitochondrial fission, fusion, and stress. Science. 2012;337(6098):1062-5.

17. Oleinik N, Kim J, Roth BM, et al., Mitochondrial protein import is regulated by p17/PERMIT to mediate lipid metabolism and cellular stress. Sci Adv, 2019; doi: 10.1126/sciadv.aax1978.

18. Martinotti S, Laforenza U, Patrone M, et al. Honey-Mediated Wound Healing: $\mathrm{H}(2) \mathrm{O}(2)$ Entry through AQP3 Determines Extracellular Ca(2+) Influx. Int J Mol Sci. 2019;20(3):764.

19. Wu B, Li J, Ni H, et al. TLR4 Activation Promotes the Progression of Experimental Autoimmune Myocarditis to Dilated Cardiomyopathy by Inducing Mitochondrial Dynamic Imbalance. Oxid Med Cell Longev. 2018. doi:10.1155/2018/3181278.

20. Liu G, Chen X, Wang Q, et al. NEK7: a potential therapy target for NLRP3-related diseases. Biosci Trends. 2020;14(2):74-82.

21. Miranda D, Jara C, Mejias S, et al. Deficient mitochondrial biogenesis in IL-2 activated NK cells correlates with impaired PGC1-alpha upregulation in elderly humans. Exp Gerontol. 2018;110:73-8.

22. Qian J, Fang D, Lu H, et al. Tanshinone IIA promotes IL2-mediated SW480 colorectal cancer cell apoptosis by triggering INF2-related mitochondrial fission and activating the Mst1-Hippo pathway. Biomed Pharmacother. 2018;108:1658-69.

23. Collins JA, Wood ST, Bolduc JA, et al. Differential peroxiredoxin hyperoxidation regulates MAP kinase signaling in human articular chondrocytes. Free Radic Biol Med. 2019;134:139-52.

24. Bogdani M, Henschel AM, Kansra S, et al. Biobreeding rat islets exhibit reduced antioxidative defense and $\mathrm{N}$-acetyl cysteine treatment delays type 1 diabetes. J Endocrinol. 2013;216(2):111-23.

25. Zhang $Z$, Wang $Y$, Shan $Y$, et al. Oroxylin A alleviates immunoparalysis of CLP mice by degrading CHOP through interacting with FBX015. Sci Rep. 2020;10(1):19272.

26. Fenech EJ, Lari F, Charles PD, et al. Interaction mapping of endoplasmic reticulum ubiquitin ligases identifies modulators of innate immune signalling. Elife. 2020. doi:10.7554/eLife.57306.

27. Pan $P$, Wang $X$, Liu D. The potential mechanism of mitochondrial dysfunction in septic cardiomyopathy. J Int Med Res. 2018;46(6):2157-69.

28. Shi C, Cai Y, Li Y, et al. Yap promotes hepatocellular carcinoma metastasis and mobilization via governing cofilin/F-actin/lamellipodium axis by regulation of JNK/Bnip3/SERCA/CaMKII pathways. Redox Biol. 2018;14:59-71.

29. Li JP, Wu H, Xing W, et al. Interleukin-27 as a negative regulator of human neutrophil function. Scand J Immunol. 2010;72(4):284-92.

30. Tournadre A, Miossec P. Interleukin-17 in inflammatory myopathies. Curr Rheumatol Rep. 2012;14(3):252-6. 
31. Kadomoto S, Izumi K, Mizokami A. The CCL20-CCR6 Axis in Cancer Progression. Int J Mol Sci. 2020;21(15):5186.

32. Gaitan-Penas H, Armand-Ugon M, Macaya A, et al. CLCN1 Myotonia congenita mutation with a variable pattern of inheritance suggests a novel mechanism of dominant myotonia. Muscle Nerve. 2018. doi:10.1002/mus.26098.

33. Rao AK, Songdej N. Parsing the repertoire of GPIb-IX-V disorders. Blood. 2017;129(4):403-4.

34. Hong T, Li LX, Han XP, et al. Effect of Astragalus membranaceus Oral Solution on Lifespan and Learning and Memory Ability of Honey Bees. Biomed Res Int. 2020. doi:10.1155/2020/5745048.

35. Lai X, Xia W, Wei J, et al. Therapeutic Effect of Astragalus Polysaccharides on Hepatocellular Carcinoma H22-Bearing Mice. Dose Response. 2017;15(1):1559325816685182.

\section{Figures}




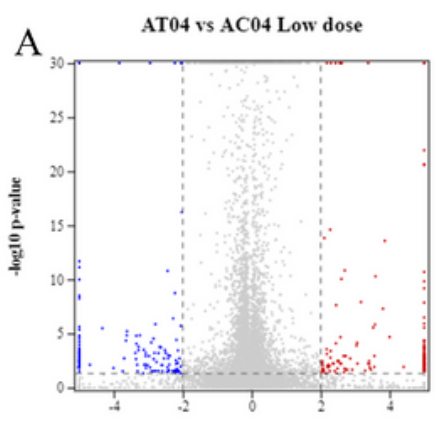

log2 Fold Change

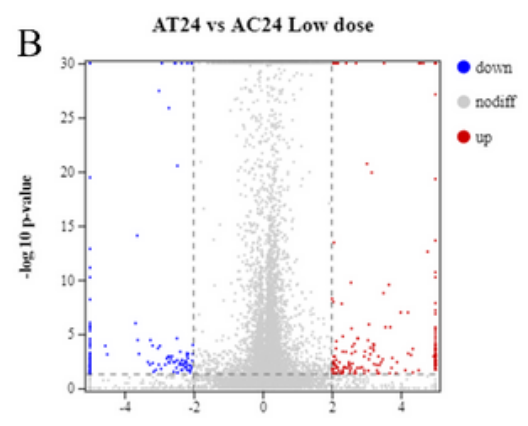

log2 Fold Change
C

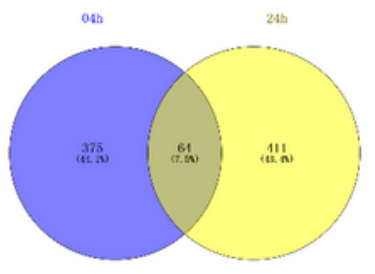

D

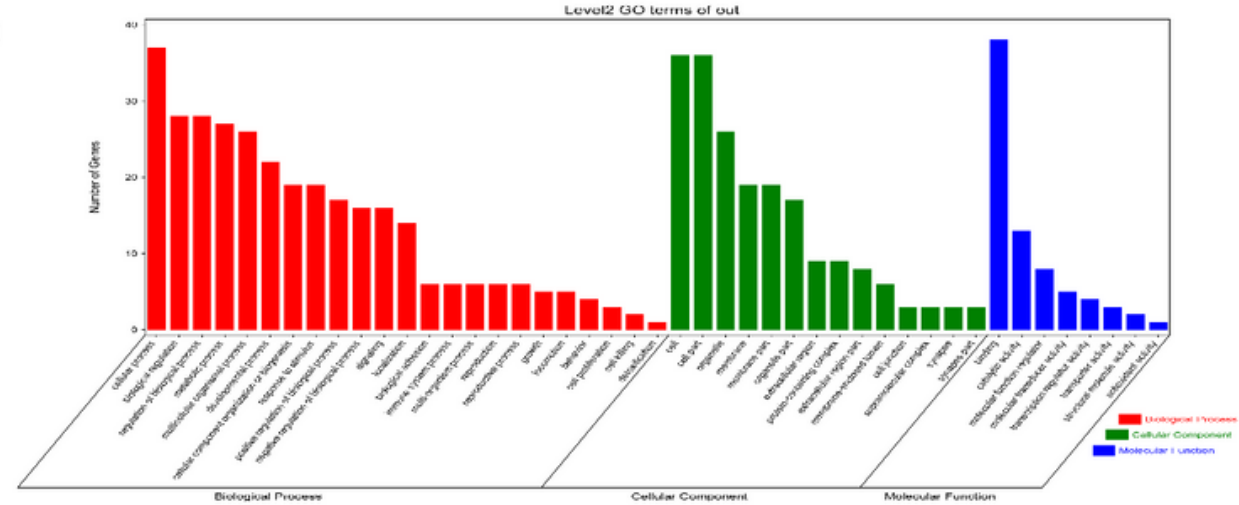

E

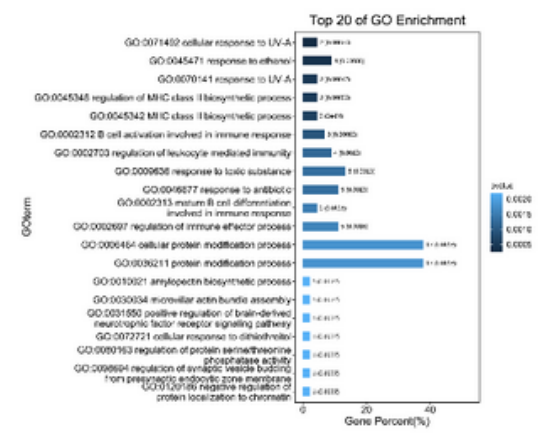

F

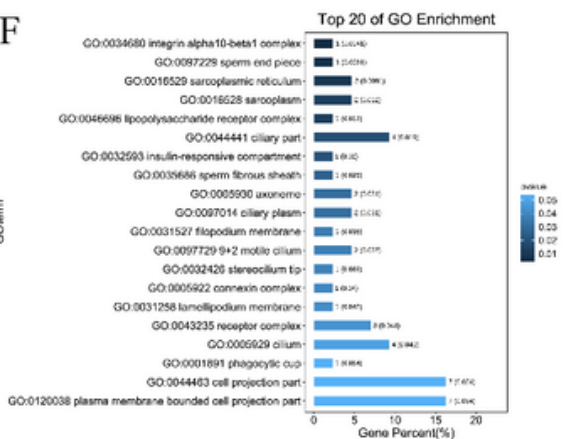

G

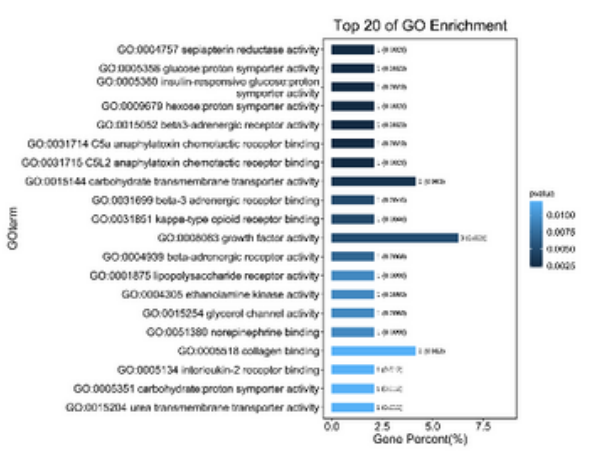

H

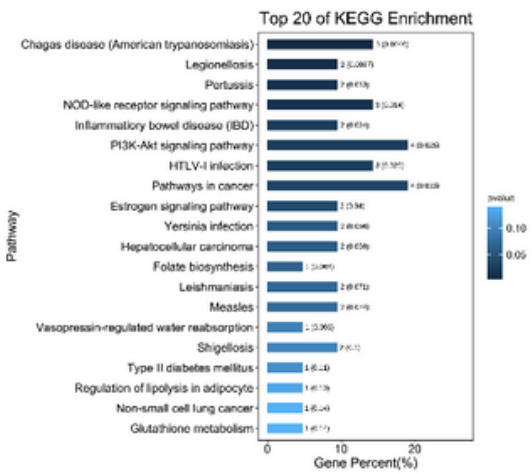

\section{Figure 1}

Co-DEGs analysis of low-dose AR extract acting in M-1 cells at different times. (A) Volcano map of DEGs between control group and treatment group at $4 \mathrm{~h}$. (B) Volcano map of DEGs between control group and treatment group at $24 \mathrm{~h}$. (C) Venn map of DEGs between $4 \mathrm{~h}$ and $24 \mathrm{~h}$ under low-dose AR extract on M-1 cell. (D) The top $20 \mathrm{GO}$ terms in the enrichment analysis. (E) The top 20 biological process (BP) terms in the enrichment analysis. (F) The top 20 cellular component (CC) terms in the enrichment analysis. (G) 
The top 20 molecular function (MF) terms in the enrichment analysis. (H) The top 20 KEGG pathway in the enrichment analysis.

A
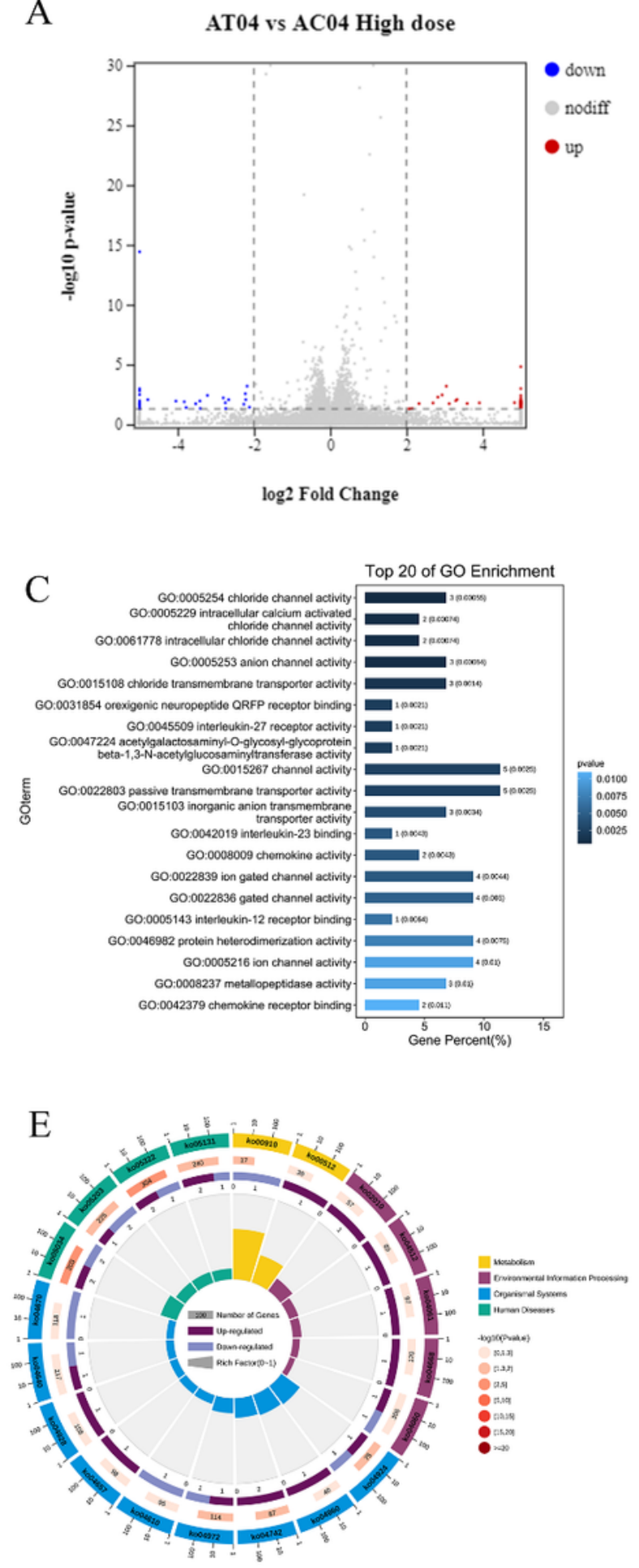

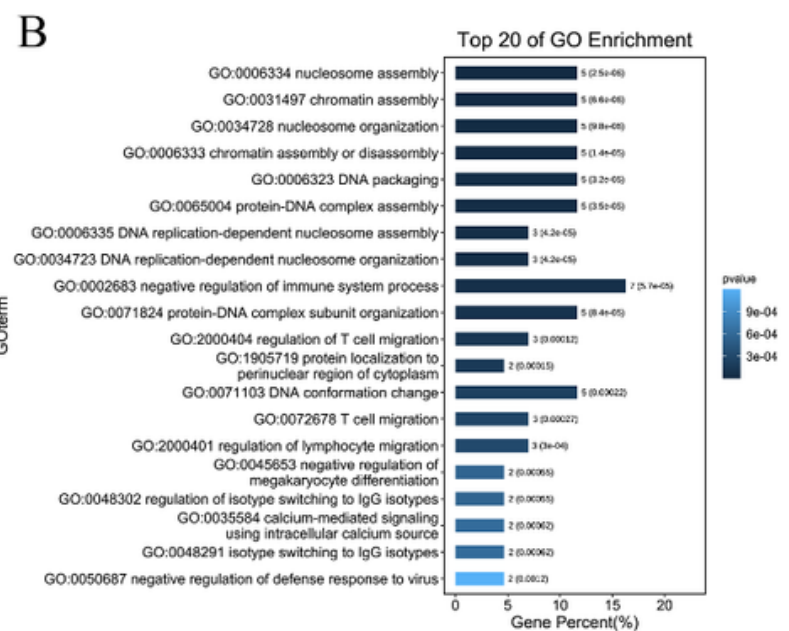

D

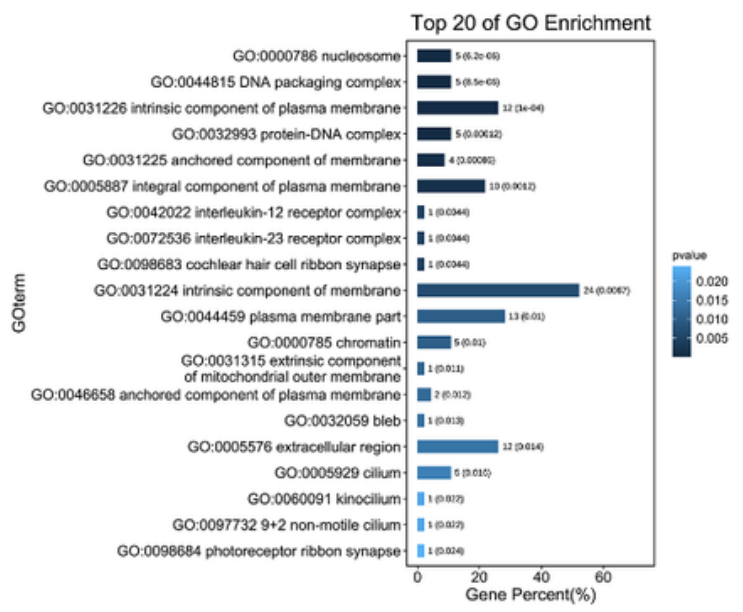

F

Low dose

High dose

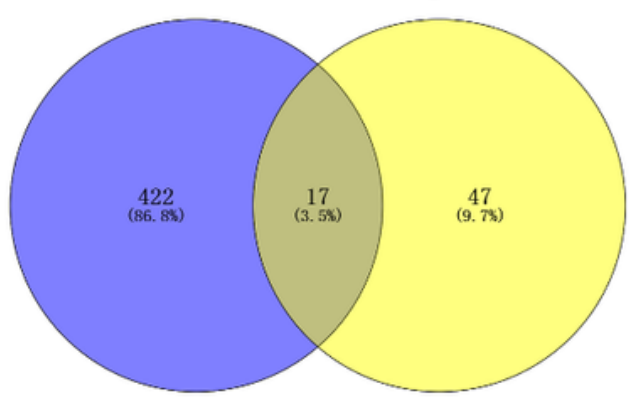

Figure 2

DEGs analysis of high-dose AR extract acting in M-1 cells at 4h. (A) Volcano map of DEGs between control group and treatment group at $4 \mathrm{~h}$. (B) The top 20 biological process (BP) terms in the enrichment analysis. (C) The top 20 molecular function (MF) terms in the enrichment analysis. (D) The top 20 cellular 
component (CC) terms in the enrichment analysis. (E) The top $20 \mathrm{KEGG}$ pathway in the enrichment analysis. (F) Venn map of DEGs between low and high dose of AR extract on M-1 cell at $4 \mathrm{~h}$. 